\title{
Supersymmetric D-brane model of space-time foam
}

\author{
John Ellis, ${ }^{1}$ Nikolaos E. Mavromatos, ${ }^{2}$ and Michael Westmuckett ${ }^{2}$ \\ ${ }^{1}$ TH Division, Physics Department, CERN, CH-1211 Geneva 23, Switzerland \\ ${ }^{2}$ Physics Department, Theoretical Physics, King's College London, Strand WC2R 2LS, United Kingdom
}

(Received 27 May 2004; published 24 August 2004)

\begin{abstract}
We present a supersymmetric model of space-time foam with two stacks of eight D8-branes with equal string tensions, separated by a single bulk dimension containing D0-brane particles that represent quantum fluctuations in the space-time foam. The ground-state configuration with static D-branes has zero vacuum energy. However, gravitons and other closed-string states propagating through the bulk may interact with the D0-particles, causing them to recoil and the vacuum energy to become non-zero. This provides a possible origin of dark energy. Recoil also distorts the background metric felt by energetic massless string states, which travel at less than the usual (low-energy) velocity of light. On the other hand, the propagation of chiral matter fields anchored on the D8-branes is not affected by such space-time foam effects.
\end{abstract}

DOI: 10.1103/PhysRevD.70.044036

PACS number(s): 04.60.-m, 11.25.Pm, 11.25.Uv

\section{INTRODUCTION}

String theory $[1,2]$ is the best candidate we have for a consistent quantum theory of gravity. Perturbative calculations yield meaningful loop corrections to scattering amplitudes, and D-branes provide a viable framework for calculating non-perturbative phenomena $[3,4]$. These are different aspects of an all-embracing $M$ theory, about which much is known even in the absence of a complete formulation.

Most studies of string or $\mathrm{M}$ theory are formulated in a fixed classical background, although it is known that the moduli space of permissible vacua is apparently very large. There has been some work on transitions between classical vacua, but we are very far from possessing an understanding of the string ground state comparable with that in, say, QCD. Just as instantons and other non-perturbative quantum fluctuations in the QCD gauge background are known to be important for understanding the infrared behavior of QCD, so we expect that the ultraviolet (and perhaps infrared) behavior of quantum gravity may only be understood when we master the physics of quantum fluctuations in the space-time background.

This space-time foam [5] might have small observable consequences even at the very low energies $E$ currently accessible to experiment. For example, there might be some breakdown of quantum coherence [6], suppressed by some power of $E$ divided by the Planck mass $M_{P} \sim 10^{19} \mathrm{GeV}$. Alternatively, there might be some deviations from the normal Lorentz-invariant dispersion relations [7-9] of elementary particles: $E \neq \sqrt{p^{2}+m^{2}}$, again suppressed by some power of $E / M_{P}$. Such Lorentz violation might not respect the principle of equivalence, in the sense that the modifications of the dispersion relations might not be universal for different particle species $[10,11]$.

The study of these possibilities requires a suitable model of space-time foam which, within string or M theory, must be based on D-brane technology [12]. As already mentioned, there is no complete theory of non-perturbative quantum fluctuations in the string vacuum. Instead, one can set up a model D-brane system possessing some features believed to be realistic. These should include having zero vacuum en- ergy in a first approximation and Planck-scale effective masses for space-time foam excitations.

In this paper we set up such a model. It is supersymmetric, with two $\mathrm{O} 8$ orientifold planes and two stacks of eight D8-branes and their mirrors, separated by a single bulk dimension in which closed strings representing gravitons are free to propagate. The bulk dimension also contains D0brane particles with effective masses related to the string scale via the inverse string coupling constant, which are of order $M_{P}$. Unlike arbitrary $\mathrm{D} p$ - $\mathrm{D} p^{\prime}$-brane configurations, this configuration has zero vacuum energy when the D8- and D0-branes are static. Open strings representing matter may attach to the D8-branes and/or the D0-particles.

We then study the interactions of matter particles with the D0-particles in this model. We find that massless closedstring states with no conventional gauge quantum numbers, such as gravitons and photons, have non-trivial interactions with the D0-particles that cause the latter to recoil $[13,14]$ with non-relativistic velocities proportional to the energies of the closed-string states. These change the vacuum energy and modify the effective space-time metric felt by the massless closed-string states $[15,12]$, in such a way as to modify their dispersion relations by corrections proportional to $E / M_{P}$. However, the open-string matter particles with internal quantum numbers do not interact with the D0-particles, so their propagation is unaffected. Thus this model of space-time foam violates the equivalence principle, in the sense that different relativistic particle species propagate differently.

In Sec. II, we review the formalism we use to compute the pertinent vacuum configurations. We then exhibit in Sec. III a consistent supersymmetric vacuum solution of string theory, involving D0-particles and two stacks of D8-branes and O8-planes. This construction has zero vacuum energy when the D0-particles are not moving, consistent with the unbroken supersymmetry of the (higher-dimensional) vacuum. There is only one bulk dimension in which closed string states such as gravitons are allowed to propagate. Open strings, on the other hand, which represent matter, may be attached to the D8-branes or the D0-particles, or stretched between them.

On the other hand, as we also show in Sec. III, when the 
D0-particle starts moving, e.g., when it acquires a non-zero velocity as a result of its recoil during its scattering with a closed-string state, there is a non-trivial vacuum (or better, excitation) energy induced on the branes. This is due to the interaction of the D8-branes with the moving D0-particle defect, and reflects the breaking of target-space supersymmetry due to the moving brane (which is a time-dependent background, from the point of view of the underlying string/ brane theory).

In Sec. IV we discuss the interaction of open string matter localized on the D-brane stack with nearby D0-particle defects, that is with defects that cross the brane or lie a string length away from it, and how these interactions affect the dispersion relation of such open string matter, which may describe low-energy gauge particles. We also discuss an extension of the above model to folded D-brane configurations, in an attempt to study the effects of the foam on the propagation of chiral matter. As in ordinary intersecting-brane cases, the latter is described by open strings localized on the (four-space-time-dimensional) brane intersection. In particular, we consider two folded stacks of D8-branes and the corresponding orientifold planes in a folded configuration, where the folding is in such a way that the folded stacks never intersect. Such a folding might have been the result of a cosmically catastrophic event, and does not cost much excitation energy. Indeed, its excitation energy relaxes asymptotically to zero at large cosmic time. As before, D0-particles populate the bulk regions. We explain that chiral matter cannot interact with these D0-particles, because such interactions are forbidden by gauge symmetries. On the other hand, string excitations that are neutral under the unbroken lowenergy gauge group (such as the photon or the graviton) may still interact non-trivially with the D0-particle defects in the supersymmetric space-time foam. This leads to "violations" of the equivalence principle, in the sense that gravity has non-universal effects on the dispersion relations of different categories of string particles.

\section{FORMALISM}

\section{A. Boundary conditions for open string}

The vacuum energy for two stacks of $\mathrm{D} p$-branes separated by a distance $R$ is described by an annulus graph, corresponding to the creation of a pair of open strings stretched between the branes. This is a pair of virtual string states, and therefore a quantum vacuum fluctuation. From a world-sheet viewpoint, one has to calculate annulus graphs in order to evaluate the force (or equivalently the potential energy) on the $\mathrm{D} p$-branes induced by such processes. Formally, if $\mathcal{A}$ denotes such an annulus amplitude, then the induced potential energy $\mathcal{V}$ on the branes can be determined by

$$
\int d t \mathcal{V}=\mathcal{A}
$$

From the point of view of a brane world, $\mathcal{V}$ may be thought of as the vacuum energy on one of the branes, in the effective theory in which the other brane is considered as an environment. Supersymmetric vacua should correspond to configu- rations for which such contributions vanish, if the D0particle and the $\mathrm{D} p$-branes are static with respect to each other. This is equivalent to a no-force condition among the branes.

There is a rich literature on the computation of such world-sheet annulus graphs, which we review in this section, for completeness, following the analysis performed by Bachas $[16,17]$.

\section{B. Mode expansion in a $\mathrm{D} p$-brane configuration}

The boundary conditions for a string stretched between two $\mathrm{D} p$-branes moving at velocities $v_{1}$ and $v_{2}$ are

$$
\begin{array}{ll}
X^{d}-v_{1} X^{0}=\partial_{\sigma}\left(v_{1} X^{d}-X^{0}\right)=0, & \sigma=0, \\
X^{d}-v_{2} X^{0}=\partial_{\sigma}\left(v_{2} X^{d}-X^{0}\right)=0, & \sigma=\pi .
\end{array}
$$

The mode expansion can be obtained in a standard way to be

$$
X^{ \pm}=\sqrt{\frac{1 \pm v_{1}}{1 \mp v_{1}}} \sum_{n} \frac{\alpha_{n}^{\nu}}{n+i \epsilon} e^{-i(n+i \epsilon)(\tau \pm \sigma)}+\text { h.c. },
$$

where

$$
\pi \epsilon=\operatorname{arctanh}(v)=\operatorname{arctanh}\left(v_{2}\right)-\operatorname{arctanh}\left(v_{1}\right) .
$$

Given the canonical commutation relations between the mode-expansion operators

$$
\left[a_{n}^{\mu}, a_{m}^{\nu}\right]=(n+i \epsilon) \delta_{n+m} \eta^{\mu \nu},
$$

we determine the Virasoro generators and thus the modification to the string Hamiltonian:

$$
\begin{aligned}
L_{0(b)}^{\|}= & \frac{Y^{2}}{4 \pi^{2} \alpha^{\prime}}+\sum_{n=1}^{\infty}(n-i \epsilon) \alpha_{-n} \alpha_{n}+\sum_{n=0}^{\infty}(n+i \epsilon) \alpha_{-n} \alpha_{n} \\
& +\frac{i \epsilon(1-i \epsilon)}{2}+L_{0}^{\perp}
\end{aligned}
$$

where $L_{0}^{\perp}$ represents the standard oscillator modes and we have performed the shift:

$$
L_{0} \rightarrow L_{0}+\frac{i \epsilon(1-i \epsilon)}{2} .
$$

A similar mode expansion can be carried out straightforwardly in the fermionic sector [17], giving

$$
\psi_{R, L}^{ \pm}=\sum_{n} d_{d}^{ \pm} \chi_{(n) R, L}^{ \pm}(\sigma, \tau),
$$

where

$$
\chi_{(n) R}^{ \pm}=\frac{1}{\sqrt{2}} \exp \left(-i(n \pm i \epsilon)(\tau-\sigma) \pm \operatorname{arctanh}\left(v_{1}\right)\right),
$$




$$
\chi_{(n) L}^{ \pm}=\frac{1}{\sqrt{2}} \exp \left(-i(n \pm i \epsilon)(\tau+\sigma) \mp \operatorname{arctanh}\left(v_{1}\right)\right),
$$

where $n=\mathbb{Z}+(a+1) / 2$ and $a=1,0$ for Ramond or NeveuSchwarz sectors, respectively. The modification to the zeromode Virasoro generator is

$$
L_{0}^{f e r m}=\sum_{n \in \mathbb{Z}+(a+1) / 2}(n \pm i \epsilon): d_{-n}^{-} d_{n}^{+}:+c(a),
$$

where

$$
\begin{aligned}
& c(0)=-\epsilon^{2} / 2, \\
& c(1)=\frac{1}{8}-\frac{i \epsilon}{2}(1-i \epsilon) .
\end{aligned}
$$

The total Hamiltonian is then

$$
L_{0}=L_{0}^{\perp}+L_{0(b)}^{\|}+L_{0}^{f e r m}
$$

where

$$
\begin{aligned}
L_{0}^{\perp}= & \mathrm{NS}\left\{\sum_{n=\mathrm{Z}}^{6-\Delta} \alpha_{-{ }_{n}} \alpha_{n}+\sum_{r=\mathrm{Z}+1 / 2}^{\Delta} \alpha_{-r} \alpha_{r}+\sum_{r=\mathrm{Z}+1 / 2}^{6-\Delta} r b_{-r} b_{r}\right. \\
& \left.+\sum_{n=\mathrm{Z}} n b_{-n} b_{n}\right\}+\mathrm{R}\left\{\sum_{n=\mathrm{Z}}^{6-\Delta} \alpha_{-{ }_{n}} \alpha_{n}+\sum_{r=\mathrm{Z}+1 / 2}^{\Delta} \alpha_{-r} \alpha_{r}\right. \\
& \left.+\sum_{n=\mathbb{Z}} n d_{-n} d_{n}+\sum_{r=\mathbb{Z}+1 / 2}^{\Delta} r d_{-r} d_{r}\right\} .
\end{aligned}
$$

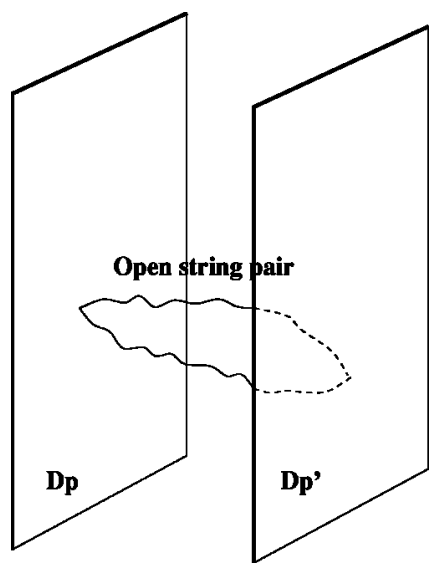

FIG. 1. Schematic representation of the open-string fluctuation between two $\mathrm{D} p$-branes. The strings are emitted from the vacuum, and occur in pairs because the chirality should be preserved.

The reason for the reduced sum over the integer modes (representing the number of $D D$ or $N N$ boundary conditions) is as follows: There would normally be $N N+D D=10-\Delta$ boundary conditions, but two are removed due to the ghost contribution which cancels two of the coordinates, leaving $8-\Delta[18]$. The modification of the $X^{0}$ and $X^{d}$ boundary conditions to include relative velocity changes them from $N N$ or $D D$ to something different, thus further reducing the sum to $6-\Delta$.

\section{Vacuum energy}

The annulus amplitude (Fig. 1) involves the trace of the $N S$ and $R$ sectors, which we separate for simplicity. The $R$ sector trace gives

$$
\begin{aligned}
\operatorname{Tr} e^{-2 \pi t\left(L_{0(b)}+L_{0}^{R}+L_{0}^{\perp}\right)=} & 2^{3-\Delta / 2} \exp \left(-8 \times 2 \pi t\left(\frac{i \epsilon}{2}(1-i \epsilon)+\frac{1}{8}-\frac{i \epsilon}{2}(1-i \epsilon)\right)\right) \frac{\left(1+e^{-2 \pi t i \epsilon}\right)}{\left(1-e^{-2 \pi t i \epsilon}\right)} \\
& \times \prod_{n=1}^{\infty}\left[\frac{\left(1+e^{-2 \pi t(n+i \epsilon)}\right)\left(1+e^{-2 \pi t(n-i \epsilon)}\right)\left(1+e^{-2 \pi t n}\right)^{6-\Delta}\left(1+e^{-2 \pi t(n-1 / 2)}\right)^{\Delta}}{\left(1-e^{-2 \pi t(n+i \epsilon)}\right)\left(1-e^{-2 \pi t(n-i \epsilon)}\right)\left(1-e^{-2 \pi t n}\right)^{6-\Delta}\left(1-e^{-2 \pi t(n-1 / 2)}\right)^{\Delta}}\right],
\end{aligned}
$$

and for the $N S$ sector we find

$$
\begin{aligned}
\operatorname{Tr} e^{-2 \pi t\left(L_{0(b)}+L_{0}^{N S}+L_{0}^{\perp}\right)}= & \frac{\exp \left(-2 \pi t\left(\frac{i \epsilon}{2}(1-i \epsilon)-\frac{\epsilon^{2}}{2}\right)\right)}{1-e^{-2 \pi t(i \epsilon)}} \\
& \times \prod_{n=1}^{\infty}\left[\frac{\left(1+e^{-2 \pi t(n+i \epsilon-1 / 2)}\right)\left(1+e^{-2 \pi t(n-i \epsilon-1 / 2)}\right)\left(1+e^{-2 \pi t(n-1 / 2)}\right)^{6-\Delta}\left(1+e^{-2 \pi t(n-1 / 2)}\right)^{\Delta}}{\left(1-e^{-2 \pi t(n+i \epsilon)}\right)\left(1-e^{-2 \pi t(n-i \epsilon)}\right)\left(1-e^{-2 \pi t n}\right)^{6-\Delta}\left(1-e^{-2 \pi t n}\right)^{\Delta}} .\right.
\end{aligned}
$$

The full amplitude is 


$$
\mathcal{V}=-2 \frac{d k_{0}}{2 \pi} \int \frac{d t}{2 t} \operatorname{Tr}\left[e^{-2 \pi \alpha^{\prime} t\left(k_{0}^{2}+M^{2}\right)}(-1)^{F_{s}} \frac{1}{2}\left(1+(-1)^{F}\right)\right],
$$

where the GSO projection results in the amplitude structure $\left[N S-R-(-1)^{F} R-(-1)^{F} N S\right]$. The total amplitude for arbitrary dimension branes with $\Delta=p^{\prime}-p$ is thus

$$
\begin{aligned}
\mathcal{V}_{D p-D p^{\prime}}= & -2 \int \frac{d t}{2 t}\left(8 \pi^{2} \alpha^{\prime} t\right)^{-1 / 2} e^{-R^{2} t /\left(2 \pi \alpha^{\prime}\right)}\left[N S-R-\delta_{(\Delta-8)}(-1)^{F} R-\delta_{\Delta}(-1)^{F} N S\right] \\
= & -\int \frac{d t}{2 t}\left(8 \pi^{2} \alpha^{\prime} t\right)^{-1 / 2} e^{-R^{2} t /\left(2 \pi \alpha^{\prime}\right)}\left[\left(1-q^{E}\right)^{-1} \prod_{n=1}^{\infty}\left(1-q^{2 n}\right)^{\Delta-6}\left(1-q^{2 n-E}\right)^{-1}\left(1-q^{2 n+E}\right)^{-1}\right. \\
& \times\left.\left(1-q^{2 n-1}\right)^{-\Delta}\right|_{B}\left\{\left.2^{\Delta / 2} q^{E / 2-1} \prod_{n=1}^{\infty}\left(1+q^{2 n-1}\right)^{6-\Delta}\left(1+q^{2 n-E-1}\right)\left(1+q^{2 n+E-1}\right)\left(1+q^{2 n}\right)^{\Delta}\right|_{N S}\right. \\
& -\left.\left.2^{4-\Delta / 2}\left(1+q^{E}\right) \prod_{n=1}^{\infty}\left(1+q^{2 n}\right)^{6-\Delta}\left(1+q^{2 n-E}\right)\left(1+q^{2 n+E}\right)\left(1+q^{2 n-1}\right)^{\Delta}\right|_{R}\right|_{(-1)^{F} R} ^{\infty} \\
& \left.-\left.\delta_{(\Delta-8)} 2^{4-\Delta / 2}\left(1-q^{E}\right) \prod_{n=1}\left(1-q^{2 n}\right)^{6-\Delta}\left(1-q^{2 n-E}\right)\left(1-q^{2 n+E}\right)\left(1-q^{2 n-1}\right)^{\Delta}\right|_{(-1)^{F} N S}\right\}_{(2.20)}
\end{aligned}
$$

where we have used the notation $q=e^{-\pi t}$ and $E=2 i \epsilon$, and $\delta_{\Delta}$ and $\delta_{8-\Delta}$ express the fact that the pertinent contribution vanishes except when the delta-function subscript is zero. A useful simplification occurs because of properties of the Jacobi theta functions, leading to

$$
\begin{aligned}
\mathcal{V}_{D p-D p^{\prime}}= & -2 \int \frac{d t}{2 t}\left(8 \pi^{2} \alpha^{\prime} t\right)^{-1 / 2} e^{-R^{2} t /\left(2 \pi \alpha^{\prime}\right)}(2 \pi)^{3-\Delta / 2}\left\{\left(\frac{\vartheta_{3}(0, q)}{\vartheta_{1}^{\prime}(0, q)}\right)^{3-\Delta / 2}\left(\frac{\vartheta_{2}(0, q)}{\vartheta_{4}(0, q)}\right)^{\Delta / 2}\left(\frac{i \vartheta_{3}(\nu t, q)}{\vartheta_{1}(\nu t, q)}\right)_{N S}\right. \\
& -\left(\frac{\vartheta_{2}(0, q)}{\vartheta_{1}^{\prime}(0, q)}\right)^{3-\Delta / 2}\left(\frac{\vartheta_{3}(0, q)}{\vartheta_{4}(0, q)}\right)^{\Delta / 2}\left(\frac{i \vartheta_{2}(\nu t, q)}{\vartheta_{1}(\nu t, q)}\right)_{R}-\delta_{(\Delta-8)}(2 \pi)_{(-1)^{F} R}^{-3+\Delta / 2} \\
& \left.-\delta_{\Delta}\left[\left(\frac{\vartheta_{4}(0, q)}{\vartheta_{1}^{\prime}(0, q)}\right)^{3-\Delta / 2}\left(\frac{\vartheta_{1}(0, q)}{\vartheta_{4}(0, q)}\right)^{\Delta / 2}\left(\frac{i \vartheta_{4}(\nu t, q)}{\vartheta_{1}(\nu t, q)}\right)\right]_{(-1)^{F} N S}\right\} .
\end{aligned}
$$

The term due to the $(-1)^{F} R$ sector is actually divergent for moving branes with $\Delta=8$ because, although the fermionic modes in the time and transverse directions no longer have zero modes (which would normally mean the sector is included), the superghosts still do [19]. This divergence is cancelled in the presence of another D8-brane, but this does not alter the velocity dependence (see below).

Using standard expansions for the theta functions, one can expand in powers of $q$, i.e., a velocity expansion for the lightest open strings. Working at the lowest power of $q$, and neglecting the bosonic kinetic terms, one has

$$
\begin{aligned}
R= & \left(2 q^{1 / 4}+2 q^{9 / 4}\right)^{3-\Delta / 2}(1+2 q)^{\Delta / 2} \\
& \times\left(2 q^{1 / 4} \cos \nu+2 q^{9 / 4} \cos (3 \nu)\right),
\end{aligned}
$$

$$
\begin{aligned}
& \qquad S=(1+2 q)^{3-\Delta / 2}\left(2 q^{1 / 4}\right)^{\Delta / 2}(1+2 q \cos 2 \nu), \\
& (-1)^{F} N S=(1-2 q)^{3-\Delta / 2}\left(\delta_{\Delta}\right)^{\Delta / 2}(1-2 q \cos (2 \nu)) \\
& \text { Combining the expansions for } N S-R \text { gives } \\
& N S-R=(1+2 q)^{3-\Delta / 2}\left(2 q^{1 / 4}\right)^{\Delta / 2}(1+2 q \cos (2 \nu)) \\
& -\left(2 q^{1 / 4}\right)^{3-\Delta / 2}(1+2 q)^{\Delta / 2}\left(2 q^{1 / 4} \cos (\nu)\right) \\
& \sim(1+(6-\Delta) q)\left(2 q^{1 / 4}\right)^{\Delta / 2}(1+2 q \cos (2 \nu)) \\
& -\left(2 q^{1 / 4}\right)^{4-\Delta / 2}(1+\Delta q) \cos (\nu)
\end{aligned}
$$

For $\Delta=0$, the $\mathcal{O}(q)$ terms are 


$$
6 q+2 q \cos (2 \nu)-16 q \cos (\nu) .
$$

Subtracting the $(-1)^{F} N S$ term

$$
\begin{aligned}
(-1)^{F} N S & =(1-2 q)^{3}(1-2 q \cos (2 \nu)) \\
& \sim-6 q-2 q \cos (2 \nu)
\end{aligned}
$$

yields

$4 q \cos (2 \nu)+12 q-16 q \cos \nu=4 q(3+\cos (2 \nu)-4 \cos (\nu))$,

so that the $\mathcal{O}(q)$ term for $\Delta=0$ is $\propto \nu^{4}$.

For $\Delta=8$,

$$
\begin{aligned}
\mathcal{O}(q) & =(1-2 q) 16 q(1+2 q \cos (2 \nu))-(1+8 q) \cos (\nu) \\
& =8 q(2-\cos (\nu))
\end{aligned}
$$

so the velocity dependence for the D0-D8 system is

$$
\mathcal{O}(q) \sim 8+4 \nu^{2} .
$$

This velocity dependence does not recover the zerovelocity limit of zero potential, because the configuration considered involves only a single D8-brane. This is because the D8-brane cannot exist on its own [3], as a result of conservation of the brane $U(1)$ flux. In the next section we present a specific construction which incorporates D8-branes and D0-particles in the bulk regions of ten-dimensional space, characterized by zero vacuum energy, thereby constituting a supersymmetric model for D0-particle foam.

\section{A SUPERSYMMETRIC DO-PARTICLE FOAM MODEL}

\section{A. Orientifold-plane(08)-D0 interaction and supersymmetric vacuum configuration}

The model we describe is based upon Type IA (alternatively Type $\mathrm{I}^{\prime}$ ) string theory, which is a nine-dimensional theory T-dual to Type I. It contains two eight-dimensional orientifold planes (O8) located at $X=0$ and $X=\pi R$, where $R$ is the radius of the compactified dimension [20]. Consistency requires that $32 \mathrm{D} 8$-branes also be present so that the -16 units of RR charge carried by each orientifold are cancelled by the D8-branes. We locate the D8-branes so that 16 lie on each O8-plane. As the D8-branes are evenly distributed, one can push the second orientifold at $\pi R$ to infinity [21]. As will be shown, this construction changes the velocity dependence of the potential at $\Delta=8$ to $\nu^{2}$, providing a supersymmetric vacuum when the velocity vanishes.

Consider the situation of a D0-brane moving toward an O8-plane (Fig. 2). The orientifold combines space-time reflection $I_{9}$ with parity reflection $\Omega$. The action of $\mathrm{O} 8$ on the oscillator mode expansions is defined as [19]

$$
\Omega I_{9}: \alpha_{n}^{1 \ldots 8} \rightarrow-(-1)^{n} \alpha_{n}^{1 \ldots 8},
$$

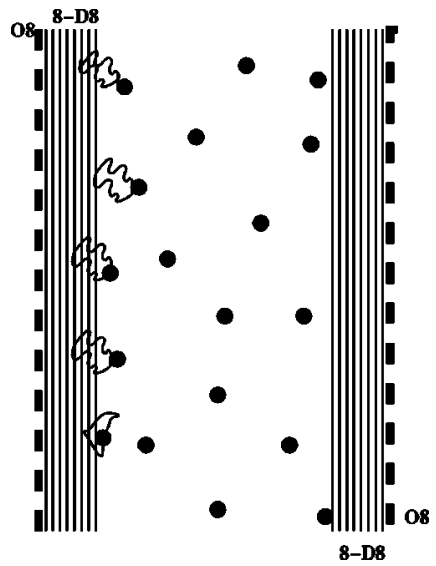

FIG. 2. A model for supersymmetric D-particle foam consisting of two stacks each of eight parallel coincident D8-branes, with orientifold planes (thick dashed lines) attached to them. The space does not extend beyond the orientifold planes. The bulk region of ten-dimensional space in which the D8-branes are embedded is punctured by D0-particles (dark blobs). The two parallel stacks are sufficiently far from each other that any Casimir contribution to the vacuum energy is negligible. Open-string interactions between D0particles and D8-branes are also depicted (wavy lines). If the D0particles are stationary, there is zero vacuum energy on the D8branes, and the configuration is a consistent supersymmetric string vacuum.

with similar action on the fermionic modes, which in terms of oscillator traces produces a shift

$$
\begin{aligned}
\Omega I_{9}\left[\left(1-q^{2 n}\right)\right] & \rightarrow\left(1+(-1)^{n} q^{2 n}\right) \\
& =\left(1+(i q)^{2 n}\right),
\end{aligned}
$$

$$
\Omega I_{9}\left[\left(1-q^{2 n-1}\right)\right] \rightarrow\left(1+(i)^{2 n-1} q^{2 n-1}\right) .
$$

A D0-brane next to an orientifold interacts with its image, as reflected by the orientifold, thus the total amplitude is a combination of half an annulus graph and half a Möbius-strip graph,

$$
\begin{aligned}
\mathcal{A}= & 2 V \int \frac{d k_{0}}{2 \pi} \int \frac{d t}{2 t} \operatorname{Tr}\left[e^{-2 \pi \alpha^{\prime} t\left(k_{0}^{2}+M^{2}\right)}(-1)^{F_{s}} \frac{1}{2}\right. \\
& \left.\times\left(1+(-1)^{F}\right) \frac{1}{2}\left(1+\Omega I_{9}\right)\right] .
\end{aligned}
$$

For a D0 brane interacting with its image, we find $\Delta=0$. There are eight possible contributions to the amplitude, corresponding to the $R$ and NS sectors with or without $(-1)^{F}$ and with or without $\Omega I_{9}$. The total expression is schematically $\left[N S-N S(-1)^{F}-R\right]+\left[-N S\left(\Omega I_{9}\right)\right.$ $\left.+N S\left((-1)^{F} \Omega I_{9}\right)+R\left((-1)^{F} \Omega I_{9}\right)\right]$ where the brackets correspond to the annulus and Möbius-strip graphs, respectively. Using the expression given before and suitably modifying for the Möbius-strip graph gives 


$$
\begin{aligned}
\mathcal{V}= & -\int \frac{d t}{4 t}\left(8 \pi^{2} \alpha^{\prime} t\right)^{-1 / 2} e^{-4 R^{2} t /\left(2 \pi \alpha^{\prime}\right)} \\
& \times(2 \pi)^{3-\Delta / 2}\left\{\left(\frac{\vartheta_{3}(0, q)}{\vartheta_{1}^{\prime}(0, q)}\right)^{3}\left(\frac{\vartheta_{3}(\nu t, q)}{\vartheta_{1}(\nu t, q)}\right)\right. \\
& -\left(\frac{\vartheta_{4}(0, q)}{\vartheta_{1}^{\prime}(0, q)}\right)^{3}\left(\frac{\vartheta_{4}(\nu t, q)}{\vartheta_{1}(\nu t, q)}\right)-\left(\frac{\vartheta_{2}(0, q)}{\vartheta_{1}^{\prime}(0, q)}\right)^{3}\left(\frac{\vartheta_{2}(\nu t, q)}{\vartheta_{1}(\nu t, q)}\right) \\
& -16\left(\left(\frac{\Theta_{4}(0, i q)}{\Theta_{1}^{\prime}(0, i q)}\right)^{3}\left(\frac{\Theta_{4}(\nu t, i q)}{\Theta_{1}(\nu t, i q)}\right)\right. \\
& -\left(\frac{\Theta_{3}(0, i q)}{\Theta_{1}^{\prime}(0, i q)}\right)^{3}\left(\frac{\Theta_{3}(\nu t, i q)}{\Theta_{1}(\nu t, i q)}\right) \\
& \left.\left.-\left(\frac{\Theta_{2}(0, i q)}{\Theta_{1}^{\prime}(0, i q)}\right)^{3}\left(\frac{\Theta_{2}(\nu t, i q)}{\Theta_{1}(\nu t, i q)}\right)\right)\right\}
\end{aligned}
$$

where the factor of 16 in the second line takes into account the difference in tension between the D-brane (annulus) and the orientifold (Möbius strip), and "twisted" versions of the Jacobi Theta functions have been used:

$$
\begin{aligned}
\Theta_{1}^{\prime}(0, i q)= & 2 \pi(i q)^{1 / 4} \prod_{n=1}^{\infty}\left(1+(i q)^{2 n}\right)^{3} \\
\Theta_{1}(\nu t, i q)= & 2(i q)^{1 / 4} \sin [\pi \nu t] \prod_{n=1}^{\infty}\left(1+(i q)^{2 n}\right) \\
& \times\left(1+(i q)^{2 n-2 \nu}\right)\left(1+(i q)^{2 n-2 \nu}\right) \\
\Theta_{2}(\nu t, i q)= & 2(i q)^{1 / 4} \cos [\pi \nu t] \prod_{n=1}^{\infty}\left(1+(i q)^{2 n}\right) \\
& \times\left(1+(i q)^{2 n-2 \nu}\right)\left(1+(i q)^{2 n+2 \nu}\right) \\
& \infty\left(1-(i q)^{2 n-1+2 \nu}\right) \\
\Theta_{3}(\nu t, i q)= & \prod_{n=1}\left(1+(i q)^{2 n}\right)\left(1+(i q)^{2 n-1-2 \nu}\right) \\
& \times\left(1+(i q)^{2 n-1+2 \nu}\right) \\
& \times\left(1+\infty \prod_{n=1}\left(1+(i q)^{2 n}\right)\left(1-(i q)^{2 n-1-2 \nu}\right)\right. \\
\Theta_{4}(\nu t, i q)= & \prod^{2 n}(3.7
\end{aligned}
$$

Expanding the theta functions for the lowest-energy string modes in terms of the velocity, we find

$$
\mathcal{V}_{\mathrm{D} 0-\mathrm{O} 8}=-2 \cos (2 \nu t)-6-120 \cos (\nu t) \sim-128+64 \nu^{2} \text {. }
$$

Taking into account the 16 D8-branes present at the orientifold, the total velocity dependence for a D0-brane moving near the $16 \mathrm{D} 8+08$ is

$$
16 \times\left(8+4 \nu^{2}\right)+\left(-128+64 \nu^{2}\right)=128 \nu^{2} .
$$

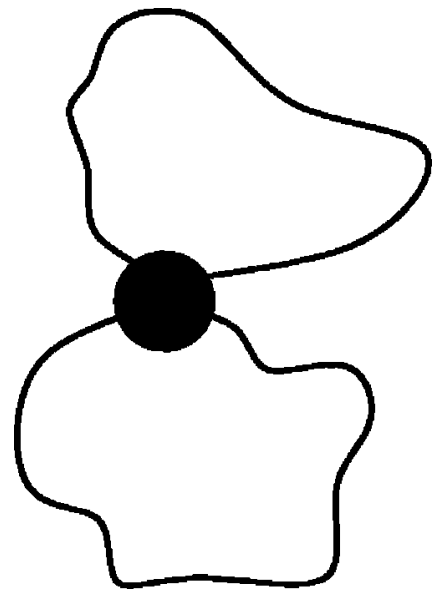

FIG. 3. The recoil of a D0-particle during scattering with a bulk closed-string state, or the spontaneous quantum fluctuation of a bulk D0-particle in the space-time foam (dark blob), is represented by a pair of open strings (wavy lines) attached to the D0-particle, if the D0-particle is isolated in the bulk.

Thus, for vanishing velocity $\mathcal{V}_{[16 \mathrm{D} 8+\mathrm{O} 8]-\mathrm{D} 0} \rightarrow 0$ as required by supersymmetry, agreeing with [22]. Therefore the above construction yields a consistent supersymmetric vacuum configuration in the zero-velocity limit. The presence of D0particle defects implies that our vacuum has a "foamy" structure. In the next subsection we consider excitations of this vacuum by giving the D0-particles a small velocity, and then study its physical properties.

\section{B. D0-particle recoil in the bulk and dark (excitation) energy on the branes}

\section{The (supersymmetric) D0-particle recoil formalism}

We have shown that D0-particles moving in the bulk space between the brane configurations will yield a non-zero potential $\mathcal{V} \sim \nu^{2}$, which can be interpreted as a contribution to the D8-brane excitation energy. From the point of view of an observer living on the D8-brane, such excitations will appear as contributions to the dark energy of the D8-brane Universe. Small velocities for the D0-particles could arise either from the scattering in the bulk of closed-string states emitted by quantum fluctuations of the D8-branes, or spontaneously, by quantum fluctuations of the D0-particles themselves, a process which also leads to the emission of closedstring states from the D0-particle, accompanied by recoil of the latter to conserve energy.

Formally, a quantum fluctuation or a recoil excitation of a supersymmetric D0-particle in the bulk ${ }^{1}$ is described by the emission of a pair of open strings with their ends attached to the D0-particle, as shown in Fig. 3. As the recoil or fluctuation happens "suddenly" at, say, time $x^{0}=0$, the corresponding $\sigma$-model deformation, describing the effects in weaklycoupled (super)strings long after the initial fluctuation, reads,

\footnotetext{
${ }^{1}$ The distinction between these processes is not important for our purposes.
} 
in the Neveu-Schwarz (NS) sector in standard world-sheet superfield notation $[14,23,24]$ :

$$
\begin{aligned}
& \mathcal{V}_{\text {rec }}=-\frac{1}{\pi} \oint d \tau d \vartheta\left(y_{i} \mathrm{C}_{\epsilon}+\nu_{i} \mathrm{D}_{\epsilon}\right) \mathcal{D}_{\perp} \mathbb{X}^{i}, \\
& \mathbb{X}^{\mu}=x^{\mu}(z)+\vartheta \psi^{\mu}(z),
\end{aligned}
$$

where $y_{i}, \nu_{i}$ are collective coordinates for the initial position and recoil velocity of the bulk D0-particle, Greek indices $\mu$ $=0,1, \ldots, 9$ are used for ten-dimensional space-time, Latin indices $i=1, \ldots, 9$ refer to spatial coordinates only, $\oint$ denotes a world-sheet boundary operator, and $\mathrm{C}_{\epsilon}, \mathrm{D}_{\epsilon}$ are chiral worldsheet superfields with components:

$$
\begin{aligned}
& C_{\epsilon}(z)=\frac{\epsilon}{4 \pi i} \int_{-\infty}^{\infty} \frac{d q}{q-i \epsilon} e^{i q x^{0}(z)}, \\
& \chi_{C_{\epsilon}}(z)=i \epsilon C_{\epsilon} \otimes \psi^{0}(z), \\
& D_{\epsilon}(z)=-\frac{1}{2 \pi i} \int_{-\infty}^{\infty} \frac{d q}{(q-i \epsilon)^{2}} e^{i q x^{0}(z)}, \\
& \chi_{D_{\epsilon}}(z)=i\left(\epsilon D_{\epsilon}(z)-\frac{2}{\epsilon} C_{\epsilon}(z)\right) \otimes \psi^{0}(z),
\end{aligned}
$$

where $\epsilon \rightarrow 0^{+}$is a regulating parameter, which is related to the ratio between the world-sheet infrared and ultraviolet cutoff scales $\Lambda / a \rightarrow 0^{+}$via $[13,14]$

$$
\epsilon^{-2}=\ln (\Lambda / a)^{2},
$$

as a result of the requirement of closure of the $N=1$ worldsheet superlogarithmic algebra. Above, the $\psi^{\mu}$ are the worldsheet superpartners of the coordinates $x^{\mu}$. The quantity $x^{0}$ denotes the temporal components in the target space, whose signature is assumed Euclidean for reasons of convergence of the world-sheet path integrals. The Minkowski signature is recovered at the end of the computation by the usual analytic continuation procedure.

Noting that

$$
\oint \frac{d q}{q-i \epsilon} e^{i q x^{0}} \propto \Theta_{\epsilon}\left(x^{0}\right)
$$

and that

$$
\oint \frac{d q}{(q-i \epsilon)^{2}} e^{i q x^{0}} \propto x^{0} \Theta_{\epsilon}\left(x^{0}\right),
$$

we observe that the bosonic parts of the recoil 'impulse' operator $\mathcal{V}_{\text {rec }}$ can be written as

$$
\mathcal{V}_{\mathrm{rec}} \ni \oint\left(\epsilon y_{i}+\nu_{i} x^{0}\right) \Theta_{\epsilon}\left(x^{0}\right) \partial_{\perp} x^{i},
$$

indicating a Galilean trajectory of the heavy D0-particle after recoil. Notice that the recoil velocity term proportional to $\nu_{i}$ is dominant in the limit $\epsilon \rightarrow 0^{+}$. It can in fact be shown [25] that the presence of this deformation at a world-sheet disc level expresses the renormalization of modular divergences in annulus diagrams during the scattering of two closedstring states with Dirichlet boundary conditions on the world sheet, corresponding to the presence of a D0-particle. This is the reason why the operator (3.11) or (3.14) describes the fluctuation of a D0-particle as shown in Fig. 3, via a pair of open strings attached to the D0-particle, whose world-sheet time evolution is described by an annulus graph, in a similar spirit to the situation discussed in the previous section. The term $\epsilon y_{i}$, which arises from the logarithmic algebra, has been interpreted in [15] as indicating an uncertainty in the initial location of the D0-particle as a result of the recoil.

It can also be shown rigorously, using the logarithmic (super)conformal algebra of the recoil operators, that momentum and energy are conserved. These conservation laws are essential $[25,15]$ for the cancellation between tree and annulus divergences mentioned above. In particular, for relative spatial momenta $k_{1,2}^{i}$ between the incident and emerging closed-string states, after recoil with the D0-particle, the requirement that the modular infinities cancel implies:

$$
\nu_{i}=M_{s}^{-1} g_{s}\left(k_{1}^{i}+k_{2}^{i}\right),
$$

yielding consistency with the following mass for the D0particle $[15,23]$ :

$$
M_{D}=\frac{M_{s}}{g_{s}},
$$

where $M_{S}$ is the string scale. In general, $M_{s}$ might be different from the four-dimensional $M_{P} \sim 10^{19} \mathrm{GeV}$. As we argue below, the latter is given by $M_{P}=\left(V^{(6)} M_{s}^{6}\right)^{1 / 2} M_{s} / g_{s}$, where $V^{(6)}$ is the compactification volume of the extra six dimensions of the string. If we assume a compactification radius of the order of $M_{s}^{-1}$, we then observe from (3.16) that the mass of the D0-particle becomes of order of the four-dimensional Planck scale, $M_{D} \sim M_{P}$.

We remind the reader that the above considerations for the recoil of the superparticle are valid in the NS sector. An extension of the superlogarithmic recoil algebra to the Ramond sector of the theory has also been made in [24], where we refer the interested reader for details. The physical conclusions relevant for our discussion in this article remain unchanged.

A final but important remark is that the recoil formalism, via the impulse operators (3.11) and (3.14), has an important difference from the scattering amplitude methods discussed so far. Although the scattering amplitude method is sufficient for calculating the contribution to the energy content of the brane, back-reaction effects of the recoiling heavy fluctuating defect on the background space-time are not accounted for by the scattering amplitude. These effects appear in the recoil formalism as a result of the fact that the recoil deformations are not conformal, but slightly relevant in a world-sheet renormalization-group sense [13].

Indeed, the world-sheet anomalous dimension of the operators (3.11) is $\Delta_{\epsilon}=-\epsilon^{2} / 2<0$. This goes to zero as $\epsilon$ 
$\rightarrow 0^{+}$, but, in view of the above-mentioned relation (3.13), there is a running anomalous dimension. In this respect, it can be shown [23] that the truly marginal coupling constants in the model are:

$$
\bar{y}_{i}=y_{i} / \epsilon, \quad \bar{\nu}_{i}=u_{i} / \epsilon .
$$

Near the infrared fixed point, we find $\epsilon \rightarrow 0^{+}\left(\ln (\Lambda / a)^{2}\right.$ $\rightarrow \infty)$. As discussed in [13], the logarithmic algebra also implies an interpretation of $\epsilon^{-2}=\ln (\Lambda / a)^{2}$ as a target time $t$, for long times after the impulse event.

This is important for calculating D-particle recoil backreaction effects in space-time. As a result of the above interpretation, for long but finite target times $M_{s}^{-1} \ll t<\infty$, the operators (3.11) are relevant in a world-sheet renormalization-group sense, and the deformed boundary $\sigma$-model theory associated with the recoil is no longer conformal. It requires Liouville dressing for consistency [26,27], since the latter operation restores the conformal invariance of the deformed theory, at the expense of introducing the Liouville mode $\phi$ into the theory as an extra world-sheet field.

It suffices for our purposes to concentrate on the bosonic part (3.14) of the boundary operator (3.11). To leading order as $\epsilon \rightarrow 0^{+}$, i.e., near the infrared world-sheet fixed point, its Liouville-dressed counterpart, $\mathcal{V}^{L}$, reads [15]:

$$
\mathcal{V}^{L} \simeq \int_{\Sigma} e^{\alpha \phi} \partial_{\beta}\left(\epsilon \bar{\nu}_{i} x^{0} \Theta_{\epsilon}\left(x^{0}\right) \partial^{\beta} x^{i}\right),
$$

where we have ignored subleading terms $\propto \epsilon^{2} \bar{y}_{i}$. Above, $\int_{\Sigma}$ denotes a world-sheet bulk integration, $\partial_{\beta}, \partial^{\beta}, \beta=1,2$ are world-sheet derivatives, and $\alpha$ is the Liouville ("gravitational") anomalous dimension. The formula (3.18) is obtained by first writing the boundary operator (3.14) as a bulk operator in the form of a total world-sheet derivative, and then dressing with the Liouville field. One can show that this is equivalent, from the physical point of view, to Liouvilledressing directly the boundary operator (3.14).

The Liouville anomalous dimension $\alpha$ is of order $\alpha \sim \epsilon$, while the corresponding central charge deficit $Q^{2}$ is supercritical $\left(Q^{2}>0\right)$ and of order $\epsilon^{4}$. This implies that the Liouville mode in the recoil problem has a Minkowski time signature [28]. Since $\epsilon^{-2}=\ln (\Lambda / a)^{2}$ is interpreted as target time, $t$, and the covariant world-sheet renormalization scale, $\ln (\Lambda / a)^{2}$, can be viewed as the zero mode, $\phi_{0}$, of the Liouville field (where the latter can be regarded as a local worldsheet scale of the non-conformal string [29]), we have proposed [30] the identification $\phi_{0} \equiv t$. Such an identification is also supported by dynamical stability arguments, related to the minimization of the effective potential energy in braneworld configurations like that encountered here [31].

Making this identification between the zero modes of the Liouville and $x^{0}$ fields in (3.18), using the representation $\Theta_{\epsilon}\left(x^{0}\right) \sim e^{-\epsilon t} \theta(t)$, where $\theta(t)$ is the ordinary Heaviside step function, and integrating by parts, we obtain, among other (boundary) terms, a bulk $\sigma$-model term of the form:

$$
\mathcal{V}^{L} \ni \int_{\Sigma} \epsilon^{2} t \theta(t) \bar{\nu}_{i} \partial_{\beta} \phi \partial^{\beta} x^{i}
$$

For long times $t \rightarrow \infty$ after the impulse event we have $\epsilon^{2} t \theta(t) \sim 1$, which implies an induced metric in target space of the form (omitting the bar notation for $\nu_{i}$ from now on, with the tacit understanding that the physical recoil velocity is given by the renormalized marginal coupling $\bar{\nu}_{i}$ ):

$$
G_{00}=-1, \quad G_{0 i} \sim \nu_{i}, \quad G_{i j}=\delta_{i j} .
$$

This expresses the back-reaction effects of the fluctuating D-particle on the neighboring target space-time. Because we used a perturbative $\sigma$-model formalism to arrive at the effect (3.20), the distortion of the target space is by definition local, and applies only in the neighborhood of the defect.

The outgoing scattered closed-string state sees the distorted space-time (3.20). From (3.15) we then observe that the magnitude of the distortion will be determined by the momentum transfer of the closed-string state during its scattering with the D0-particle. In general, this affects the dispersion relation of the scattered string state, by an amount depending on the density of D0-particles in the bulk. We return later to this important issue, in particular in connection with the possible modification of the dispersion relation of (observable) matter localized on the brane.

\section{Contributions to the dark energy of the brane world}

We now estimate the dark energy in the brane world on the basis of the above analysis. Let $n_{0}$ be the density of the D0-particles in the ten-dimensional bulk space, which we assume to be uniform. We also assume that the exchanged pairs of strings between the D0-particles and the D8-branes cannot be stretched too long, so that only D0-particles close to the branes, i.e., within a distance of order $\ell_{s}$, can be "felt" by the D-brane world and make significant contributions to the brane "dark" (excitation) energy, We also assume that the "gas" of D0-particles is sufficiently dilute that interactions among the D0-particles can be safely ignored.

The recoiling defects near the D8-brane world will in general have a distribution of velocities $\mathcal{P}\left(\nu_{i}\right)$. To simplify things, we assume a uniform average velocity for the relevant D0-particles, which is a valid approximation if the velocities are small, the case of relevance to us here. We stress that, for a recoiling defect near the brane worlds, there are two effects to be considered: (i) the recoil itself, described by the string pair fluctuations of Fig. 3 and given formally by the deformation (3.11), which is responsible for a non-zero velocity $\nu_{i}$ for the defect, and (ii) the exchange of open strings between the D0-defect and the D8-brane world, described by the annulus graphs of Fig. 1 in the case where the brane is a D0-particle. It is the latter effect that yields the amplitude $\mathcal{A}$ contributing to the dark energy of the brane world, while it is the former effect that yields the velocity of the D0-particle, and hence contributes terms of order $\nu^{2}$ to the amplitude [cf. (2.28)].

Assuming that the extra five longitudinal dimensions of the D8-branes are compactified on a manifold with volume $V^{(5)}$, the total contribution of the induced dark energy on the branes can be determined from (2.1) to be 


$$
\int d t V_{\text {brane, total }}=\int_{\text {eff }} d^{(10)} x n_{0} \mathcal{A} \sim \int_{\text {eff }} d y d^{(4)} x n_{0} V^{(5)} \nu^{2}
$$

where $\int_{\text {eff }}$ denotes the "effective" bulk volume in the immediate vicinity of the brane complex, over which the presence of the D0-particles makes significant contributions to the dark energy on the brane, $y$ denotes the bulk coordinate along the ninth dimension and $x$ the four-dimensional spacetime coordinates, and we used the results of the previous subsection to set $\mathcal{A} \sim \nu^{2}$. $^{2}$

For the sake of definiteness, we assume that we have an effective bulk dimension of size $R_{9} \sim \ell_{s}=M_{s}^{-1}$, where $\ell_{s}\left(M_{s}\right)$ is the string length (mass) scale, which may, in general, be different from the four-dimensional Planck scale $\ell_{P}=10^{-33} \mathrm{~cm}\left(M_{P} \sim 10^{19} \mathrm{GeV}\right)$. The two scales are related through [32]

$$
M_{P} \sim\left(M_{s}^{6} V^{(6)}\right)^{1 / 2} g_{s}^{-1} M_{s},
$$

where $g_{s}$ is the string coupling, which is assumed in this work to be weak: $g_{s}<1$, so that perturbative string theory, i.e., a $\sigma$-model analysis, is a valid approximation. If the compactified dimensions are of the same order as the string scale, as assumed for definiteness here, then we have $M_{P}$ $=M_{s} / g_{s}$. More complicated relations can occur, however, as the sizes of the various extra dimensions may vary. In our generic analysis below we do not discuss such cases, although the extension of our analysis is straightforward.

Performing the integration over $y$ in (3.21), assuming a uniform bulk distribution of the "effective" D0-particles, we then obtain the following contribution to the fourdimensional dark energy:

$$
\Lambda^{(4)}=R_{9} n_{0} \nu^{2} V^{(5)} \sim \ell_{s}^{6} n_{0} \nu^{2},
$$

assuming for definiteness a compactification radius of order of $\ell_{s}$. The current observational limits on the fourdimensional dark energy imply $\Lambda^{(4)}<10^{-123} M_{P}^{4}$, from which we obtain an upper limit on the density of the "effective" D0-particles of

$$
\widehat{n_{0}} \equiv \ell_{s}^{10} n_{0}<10^{-123} \nu^{-2} g_{s}^{-4}
$$

defects per ten-dimensional string volume.

We now estimate $\nu^{2}$ in our framework. Energy conservation in the recoil problem of closed-string states scattering off a heavy non-relativistic D0-brane defect $[23,14]$ implies that

$$
\nu^{2} \sim(\Delta k)^{2} / M_{s}^{2},
$$

where $\Delta k$ is the momentum transfer during the collision and $M_{s}$ is the string scale. The magnitude of this depends on the

\footnotetext{
${ }^{2}$ Note that $R_{9}$ should not be confused with the distance $r_{0}$ to the location of the other parallel stack of D8-branes, which are assumed to lie far enough away that any Casimir energy contribution to the energy is negligible.
}

details of the microscopic bulk string theory. We may assume as typical energies of bulk graviton states those given by temperature effects in the bulk Universe. This could be very low, as one may quite naturally assume that the two stacks of D8-branes that constitute our vacuum have collided at some early stage, and are now moving with very slow velocities, in such a way that they are now almost static, and sufficiently far away from each other in the bulk. In this case, the interaction rates of closed-string states in the bulk are very suppressed, and the bulk string temperature can be naturally very low. On the other hand, on the brane world, where open string matter is confined, the matter interaction rates may be assumed to be significantly larger, which will lead to larger brane temperatures as compared to that in the bulk. Hence, in this scenario, at the present era the brane Universe is much hotter than the bulk region, and the configuration has not reached thermodynamic equilibrium. As the brane universe continues to expand, the brane temperature decreases at an ever-decreasing rate, whilst the rate of decrease of the bulk temperature may be assumed to have vanished already at some bulk freeze-out temperature. This means that eventually thermodynamic equilibrium between the brane and the bulk will be achieved. In this scenario, therefore, the fourdimensional dark energy density on the brane, given by (3.23), is not constant but relaxes to zero at a rate which is determined by the temperature relaxation in the bulk region (assuming an almost constant bulk density of D0-particles in the ten-dimensional space today). This, as we have remarked above, can be very small and for all practical purposes undetectable.

We now consider some numbers, so as to understand better the need for two different temperatures between bulk and branes. We first consider the case that, at the present era, the brane universe is already at thermal equilibrium with the bulk region. Since today we have microwave background temperatures on the brane worlds of about $2 \mathrm{~K}$, this yields a typical momentum transfer for bulk gravitons

$$
\Delta k \sim k \sim \mathcal{O}(K) \sim \mathcal{O}\left(10^{-13} \mathrm{GeV}\right) \sim 10^{-32} M_{s} / g_{s},
$$

where we used (3.22), that is $M_{P}=M_{s} / g_{s}$. This yields the following average for $\nu^{2}$ :

$$
\left\langle\nu^{2}\right\rangle \sim 10^{-64} g_{s}^{-2},
$$

in natural units where the speed of light in vacuo $c=1$. This implies a gas of D0-particles with $\widehat{n_{0}}<10^{-59} g_{s}^{-2}$ defects per ten-dimensional string volume. In string theory $g_{s}$ can be as low as $10^{-14}$ in theories with large extra dimensions, where the string scale can be as low as a few hundreds of $\mathrm{TeV}$ for phenomenologically acceptable models [32]. In this case, the current dark energy content of the observable Universe would require a very dilute gas of D0-particles with $\widehat{n_{0}}$ $\sim 10^{-31}$ defects per ten-dimensional string volume.

Much larger values of $\widehat{n_{0}}$ can be obtained if one assumes a much smaller bulk temperature, a case which, as discussed above, can easily be accommodated in physically meaningful situations. For instance, in order to obtain natural models of 
D0-particle foam in ten dimensions, with $\widehat{n_{0}} \sim O(1)$ defects per ten-dimensional string volume, we see from (3.24) that one must have

$$
\left\langle\nu^{2}\right\rangle \sim 10^{-67} \text { for } g_{s} \sim 10^{-14} .
$$

The above thermal considerations would then imply a bulk temperature $T_{\text {bulk }} \sim 3 \times 10^{-16} \mathrm{~K}$, i.e., sixteen orders of magnitude smaller than the current temperature in our D-brane Universe.

In such situations, the relatively "hot" (compared to the bulk) Universe will radiate heat into the bulk in the form of the kinetic energy of closed-string states emitted from the brane into the bulk. The process by which closed strings radiate from a D-brane can be described by a three-point tree-level process, where two open strings attached to the brane collide and form a closed string which moves off into the bulk [33]. In combination with the pair-production rate for open strings, this would, in principle, enable one to calculate the temperature of the bulk due to emitted closed strings. It is expected that in situations where the fourdimensional Planck length is much smaller than the string scale this rate is very low, probably undetectable at present for all practical purposes.

\section{MATTER PROPAGATION IN THE D-BRANE FOAM MODEL}

\section{A. Modified dispersion relations for massless gauge excitations}

We now discuss some important physical consequences of the presence of D0-particles near the brane world, namely possible modifications of the dispersion relations for certain string matter states propagating on the brane world. We first consider gauge degrees of freedom represented by open strings with their ends attached on the brane. Because such matter cannot propagate in the bulk, the only possible interaction of the string with a D0-particle is that represented in Fig. 4. The D0-particles that interact with the string are sufficiently close to the brane (within the uncertainty string scale $\ell_{s}$ ) that they might be considered as practically "on" the brane worlds when one considers the fluctuations of the branes to be expected in any consistent quantum-gravity model of branes.

We discuss two effects that appear in situations like that in Fig. 4. (i) The contribution of the process shown in Fig. 4 to the excitation energy of the brane. This process involves the splitting of an open string with its ends attached to the D-brane into two open strings, each with one end attached to the D0-particle, and the other to the D-brane. Each length of string corresponds to an interaction described by a tree-level world-sheet amplitude with appropriate Dirichlet boundary conditions. As the open strings here represent excitations of the brane world, and not quantum fluctuations as in Fig. 1, the corresponding amplitude should be viewed as a fluctuation in the energy of the open string and not as a contribution to the vacuum energy on the brane. (ii) The other effect is the recoil of the D0-particle itself, as a result of momentum transfer from the string to the D0-particle, in accord with energy-momentum conservation. This has been described in

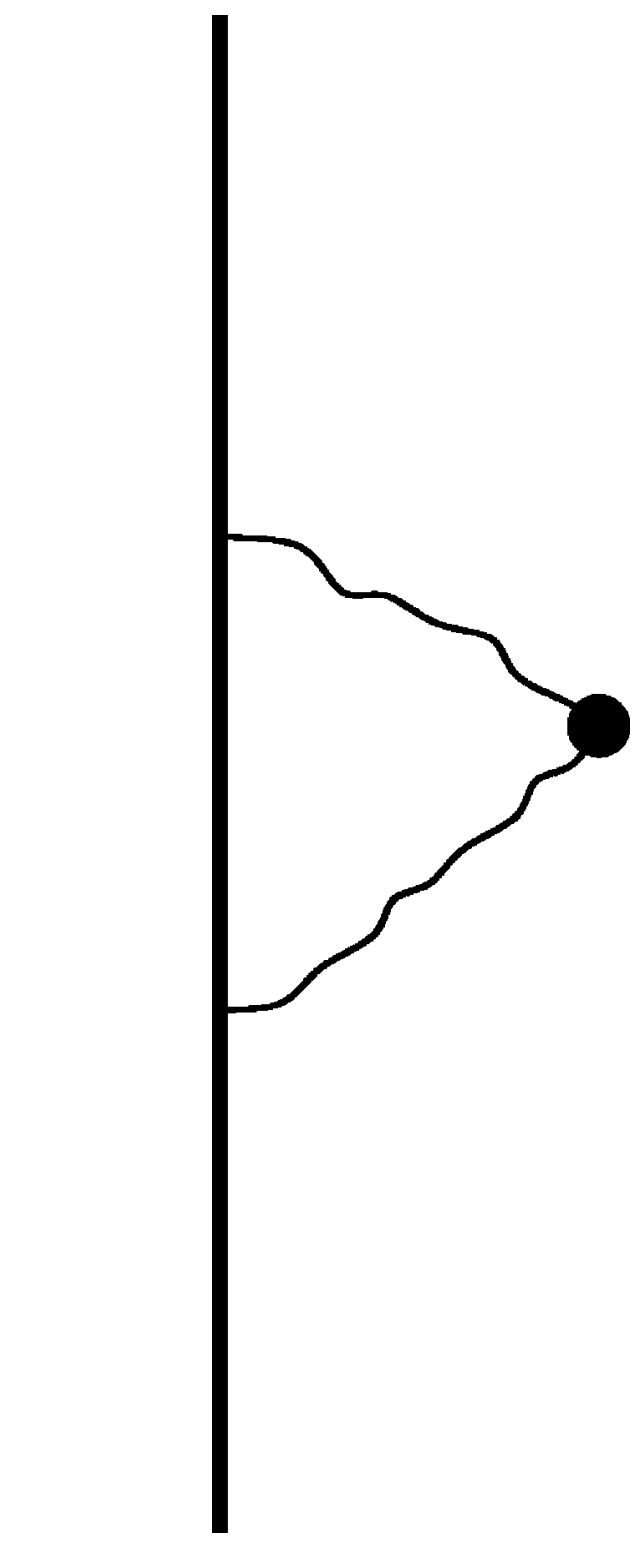

FIG. 4. The interaction of an open string propagating on the brane with a D0-particle defect that crosses the brane, or is at a small distance $\sim \ell_{s}$ away from it, implies the splitting of the initial string into two strings stretched between the D-particle and the brane.

previous subsections, and is responsible for the distortion (3.20) of the space-time near the recoiling defect.

The recoil formalism makes possible the computation of the modifications of dispersion relations, i.e., local (meanfield) relations between energy and momentum, for open string states propagating on the brane that result from their interactions with a population of defects. We recall that, after scattering, an open-string state will propagate on the distorted space-time (3.20). Let $p_{\mu}$ denote the four-momentum of the low-energy particle excitation corresponding to a particular vibrationary mode of the open string. This is a massless gauge particle, as the open string represents a gauge excitation in the stack of D8-branes, transforming in an adjoint representation of the $U(8)$ group for a stack of eight D8-branes [34]. 
The dispersion relation of the scattered gauge particle in the distorted space-time $G_{\mu \nu}$ (3.20) in the neighborhood of the defect reads: $p_{\mu} p_{\nu} G^{\mu \nu}=0$, from which we obtain $E^{2}$ $-2 E p_{i} \nu^{i}-p_{i} p^{i}=0$. The positive energy solution $E>0$ that connects smoothly with the case of no recoil $\nu_{i} \rightarrow 0$ is

$$
E=\boldsymbol{p} \cdot \boldsymbol{\nu}+|\boldsymbol{p}|\left(1+\left(\boldsymbol{\nu} \cdot \frac{\boldsymbol{p}}{|\boldsymbol{p}|}\right)^{2}\right)^{1 / 2},
$$

where the notation $\boldsymbol{a}$ denotes a nine-dimensional spatial vector, $\boldsymbol{p}$ is the momentum of the outgoing (open) string excitation, and $\boldsymbol{\nu}$ is the recoil velocity of the D0-particle. The dispersion relation (4.1) also applies to closed-string states, such as particle excitations in the gravitational multiplet, due to scattering off defects in the bulk.

There are several implications for the dispersion relations of $U(8)$ gauge particles (4.1) derived above, in the context of our stringy supersymmetric foam model: First, we remark that the group velocity of the massless gauge particles is $v_{g}$ $=\partial E / \partial p$, with $p \equiv|\boldsymbol{p}|$. The recoil velocity is connected to the momentum transfer during the interaction via momentum conservation (3.15). It is natural to assume $M_{D}|\boldsymbol{\nu}| \sim p$, i.e., the maximum magnitude of the momentum transfer is of the same order as the outgoing (or incident) momentum. One should average the relation (4.1) over the D0-particles in the foam, and we denote such an average by $\langle\langle\cdots\rangle\rangle$. We recall that, in our conventions, $\left\langle\left\langle\nu^{i}\right\rangle\right\rangle=\left\langle\left\langle k_{\text {out }}^{i}+k_{\text {in }}^{i}\right\rangle\right\rangle$ denotes the average momentum transfer. The sign of $\left\langle\left\langle\nu^{i}\right\rangle\right\rangle$ depends on whether the interaction with the foam defect accelerates or slows down the particle. We expect on average that there should be anisotropy in the direction of the average recoil velocity of the foam D0-particle, oriented along that of the propagating incident particle [35].

String theory leads to an unambiguous specification of the sign of $\left\langle\left\langle\nu^{i}\right\rangle\right\rangle$, since in a string theory spectrum there should be no superluminal particles. Indeed, the dynamics of the foam defects themselves has a Born-Infeld form, leading to subluminal velocities. From (4.1) we observe that subluminal propagation requires an average deceleration of the particle by the foam, $\langle\langle\boldsymbol{\nu} \cdot \boldsymbol{p}\rangle\rangle=-\langle\langle|\boldsymbol{\nu}| p\rangle\rangle<0$ (in our conventions), leading to group velocities suppressed minimally by a single power of $M_{P} \sim M_{s} / g_{s}$ :

$$
v_{g}^{\text {capture }} \sim 1-\left|\mathcal{O}\left(p / M_{P}\right)\right|, \quad M_{P} \sim M_{s} / g_{s} .
$$

In contrast, we notice that, in isotropic models of D0-particle foam for which $\langle\langle\boldsymbol{\nu}\rangle\rangle=0$, one would obtain from (4.1) a superluminal (as compared to the speed of light in the no-recoil vacuum) average group velocity for massless gauge particles with quadratic suppression in $M_{P}^{-1}:\left\langle\left\langle v_{g}\right\rangle\right\rangle$ $\sim\left\langle\simeq 1+\mathcal{O}\left(p^{2} / M_{P}^{2}\right)>1\right.$. Such models are therefore not consistent with string theory.

\section{B. Chiral matter in the foamy brane model: intersecting branes}

We now discuss whether chiral matter in brane models can have analogous interactions with D-particle defects in the foam. Chiral matter in models involving stacks of
D-branes is described by open-string excitations localized at intersections of the brane configurations. If there is no intersection, then the open-string excitations of the brane worlds describe only gauge particles. This remains true in the presence of impulse operators of D0-particles, given that, as shown in detail in [23], the dynamics of such fluctuating D0-particles (or coincident groups of D0-particles interacting via the exchanges of strings, or D0-particles near brane worlds) are described by a (non-Abelian) Born-Infeld gauge action. Therefore, such excitations behave as gauge excitations, transforming in the adjoint representations of $U(N)$ groups, for a stack of $N$ branes or a coincident group of $N$ particles.

A natural question to ask at this point concerns the precise gauge group which arises in the case of a D-particle sufficiently close (i.e., within a string length $\ell_{s}$ ) to a stack of $\mathrm{N}$-coincident branes and a real open string excitation stretching between the D-particle and the stack. Since the open string cannot have both of its ends off brane (not even within an $\ell_{s}$ length away; by definition at least one of them must be attached to the brane), then by a simple counting argument of the available states, that is of the available ways the other end of the string is attached to the various members of the D-brane stack, one obtains a $U(N)$ group in this case, instead of the $U(N+1)$ which would arise if both ends of the open string could be attached to the D-particle.

To obtain chiral matter, we need to consider intersecting branes [36] at an angle $\theta \neq 0$. In a situation where one has an intersection of a stack of $N$ parallel D-branes with another stack of $M$ parallel D-branes, the chiral matter is in the fundamental representation of the $U(N) \otimes U(M)$ group, while gauge matter (also represented by open strings on the intersection) falls into the adjoint representation of the $U(N)$ $\otimes U(M)$ gauge group. On the other hand, open strings attached on the individual stacks far away from the intersection fall into adjoint representations of the $U(N)$ or $U(M)$ gauge group, depending on which stack they are attached to. Of course, at the intersection one has also gauge excitations (open strings). Before proceeding toward a precise construction of brane configurations allowing for chiral matter in the context of our D-particle foam model, it is useful to make some remarks on the general problem of D-brane stacks and D0-particles, which clarifies some important properties of our supersymmetric vacuum construction.

\section{A model for chiral matter in supersymmetric D-foam}

In the presence of orientifold planes, beyond which the space cannot be extended because of their reflection properties, the normally straightforward scenario of intersecting branes becomes more complicated. On the other hand, there is the case of brane folding, which may be the result of a catastrophic collision between two branes of different dimensionality [37]. The folding of D-branes corresponds to an excitation of the vacuum, and in a world-sheet framework it may be described by appropriate logarithmic operators,

$$
\mathcal{V}_{\text {fold }}=g_{I i} \Theta \Theta_{\epsilon}\left(X^{I}\right) X^{I} \partial_{n} X^{i},
$$

where $X^{I}$ is a longitudinal coordinate of the D-brane, $X^{i}$ is a 
transverse coordinate, $\partial_{n}$ denotes normal world-sheet derivative, and the $\sigma$-model coupling $g_{I i}$ describes the characteristics of folding, that is the tangent of the angle at the folding region, i.e., the brane intersection.

The departure from the conformal point due to the logarithmic operators describes the excitation of the folded brane. A simple world-sheet renormalization group analysis shows that the folding operators (4.3), like the corresponding D-particle recoil operators (3.11), (3.12), are relevant operators with anomalous dimension $-\epsilon^{2} / 2, \epsilon \rightarrow 0^{+}$. We identify $\epsilon^{-2}$ with the logarithmic renormalization-group scale $\epsilon^{-2}$ $=\ln \Lambda \equiv \mathcal{T}$, on account of the requirement of closure of an appropriate logarithmic algebra [13]. To estimate the foldinginduced brane excitation energy one needs to compute the renomalization-scale dependent ("running") induced central-charge deficit. The latter is evaluated by means of Zamolodchikov's $c$-theorem, which gives the rate of change of the "running" central charge $Q^{2}(\mathcal{T})$ of this relevant (nonconformal) deformed $\sigma$-model theory,

$$
\frac{d}{d \mathcal{T}} Q^{2}=-\beta^{i} \mathcal{G}_{i j} \beta^{j}=-\frac{\bar{g}_{I i}^{2}}{\mathcal{T}^{2}},
$$

where $\beta^{i}$ is the renormalization-group $\beta$ function of the appropriate coupling $g^{i}$ corresponding to the vertex operators $\mathcal{V}$ of folding (4.3), $\mathcal{G}_{i j} \sim|z|^{4}\left\langle V_{i}(z) V_{j}(0)\right\rangle$ is the Zamolodchikov metric in theory space, and we keep only the dominant terms near the infrared fixed point $($ as $\mathcal{T} \rightarrow \infty)$. Above, $\bar{g}_{I i}=g_{I i} / \epsilon$ is a scale $(\epsilon)$-independent "renormalized" coupling. Equation (4.4) shows that the resulting excitation energy on the brane due to folding is of order:

$$
Q^{2}(\mathcal{T}) \sim Q_{0}^{2}+\frac{\bar{g}_{I i}^{2}}{\mathcal{T}}
$$

In our case we have $Q_{0}=0$, since as shown above, in the absence of brane folding one has a supersymmetric vacuum string configuration, characterized by zero central-charge deficit. The non-conformal $\sigma$-model becomes conformal by Liouville dressing, and we identify the world-sheet Liouville zero mode $\varphi_{0}$ with $\mathcal{T}$, since we view the Liouville mode as a local dynamical renormalization scale on the world sheet.

It is customary [26,31] to normalize the kinetic term of the Liouville action by redefining $\varphi \rightarrow \phi=Q(\mathcal{T}) \varphi$, which plays the role of an extra target space-time dimension. In our case (4.5) the deformed string is supercritical $Q^{2}>0$, and the Liouville mode has time-like signature in target space.

In view of this rescaling we may then write (4.5) as

$$
Q^{2}=\frac{1}{\phi_{0}^{2}}\left(\bar{g}_{I i}^{2}\right)^{2},
$$

where $\phi_{0}$ is the world-sheet zero mode of the rescaled Liouville field. If we identify the Liouville mode with target time, ${ }^{3} t$, then the one obtains relaxation to zero, where the excitation energy due to bending relaxes to zero as $1 / t^{2}$. In

\footnotetext{
${ }^{3}$ Dynamical arguments for this identification, related to the minimization of appropriate potentials of the effective low-energy theory, may be provided in some cases, see [31].
}

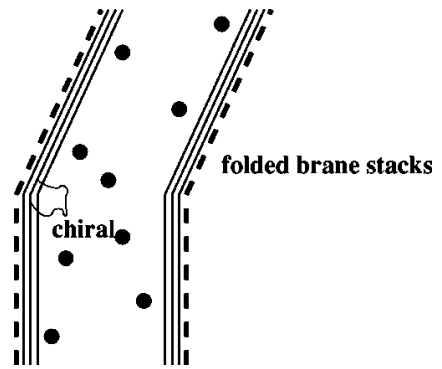

FIG. 5. A model consisting of folded parallel pairs of stuck of D8-branes with orientifold planes (thick dashed lines) attached to them. The folding might have been induced by a catastrophic cosmic event. The bulk region of the ten-dimensional space in which the branes are embedded is punctured by D0-particles (dark blobs). The space is not extended beyond the orientifold planes. The branes intersect at an angle $\theta$, and chiral matter is described by open strings localized on the seven-dimensional intersections. For clarity, we do not depict the open string interactions between D0-particles and branes.

this way one has a unified picture between D0-particle recoil and the folding of branes in this foam model. This picture seems to describe an elastic brane situation where the folding process can eventually disappear at long times after the initial catastrophic event, leading to an equilibrium configuration.

In our construction, which necessarily involves an auxiliary stack of branes at infinity, it appears necessary to bend this auxiliary stack in the same direction, so intersection between the two brane stacks is avoided. Thus, the construction depicted in Fig. 5 may yield an asymptotically supersymmetric vacuum in the absence of any movement or quantum fluctuations of the D0-particles in the foam.

The model consists of the supersymmetric D-foam model considered in Sec. III, with one of its branes folded at some region, so that an angle $\theta \neq 0$ is created in the bulk tendimensional space. Both the branes and its images are folded in the same way, so that any intersections between the two stacks of branes are avoided. The distances between the observable world and its images in this supersymmetric foam model are very large, so that any Casimir contribution to the vacuum energy can be ignored. The observable world in which we live is the resulting seven-dimensional intersection of the D8-branes with the four extra spatial dimensions compactified. Chiral matter is localized on this intersection, and D0-particles puncture the bulk regions, yielding the foam effects described above. Due to the arguments on folding presented above, the uncompactified model has a vacuum energy that almost vanishes for large times after the folding, $t \rightarrow \infty$, in the no-recoil limit of the D0-particles.

Chiral matter cannot interact non-trivially with D0particles near the intersection, i.e., at distances within the uncertainty limit $\ell-s$, as gauge particles did. The reason is that the splitting of a chiral matter string by the defect, in analogy with Fig. 4, would result in a situation in which the D0-particle would exchange open strings with the intersecting stacks of D8-branes. Each D0-D8 interaction would find itself in states that would fall into the adjoint representation 
of the $U(8) \otimes U(8)^{\prime}$ gauge group, since the open strings must always have at least one of their ends attached on the intersection. This would always be a gauge excitation, and hence the process of Fig. 4 would not be allowed for chiral matter at the intersection of the brane stack, being prevented by the unbroken gauge symmetry. One may understand this last property from a more physical point of view by considering the fact that a D0-particle has no spatial extent, so it is not possible to define an angle with the D-brane. In this sense, a D0-particle is always "parallel" to the D-brane, and crosses it effectively at a zero angle. Hence, chirality in the sense of [36] cannot be defined for such open-string configurations stretching between the defect and the brane when they are split by a D0-particle.
This implies that our supersymmetric D-brane foam violates the universality of gravitational effects on matter, and in this sense the equivalence principle, a scenario advocated already in $[10,11,38]$. This is important, in view of the stringent constraints on linear modifications of dispersion relation for chiral matter (in particular electrons and other charged fermions), which do not apply to photons. On the other hand, present limits on photons and gauge particles cannot yet exclude linear suppression effects in their dispersion relations. The most stringent limit on subluminal photon dispersion relations can be derived from studies of the arrival times of gamma-ray bursts $[8,39,40]$, from which the effective gravity scale entering the linear modification of the photon dispersion relation is found to be bounded by $M_{Q G}>10^{16} \mathrm{GeV}$.
[1] M. B. Green, J. H. Schwarz, and E. Witten, Loop Amplitudes, Anomalies and Phenomenology, Superstring Theory Vol. 2 (Cambridge University Press, Cambridge, 1987), p. 596 (Cambridge Monographs On Mathematical Physics).

[2] M. B. Green, J. H. Schwarz, and E. Witten, Introduction, Superstring Theory Vol. 1 (Cambridge University Press, Cambridge, 1987), p. 469 (Cambridge Monographs On Mathematical Physics).

[3] J. Polchinski, Superstring Theory and Beyond, String Theory Vol. 2 (Cambridge University Press, Cambridge, 1998), p. 531.

[4] J. Polchinski, An Introduction to the Bosonic String, String Theory Vol. 1 (Cambridge University Press, Cambridge, 1998), p. 402.

[5] J. A. Wheeler and K. Ford, Geons, Black Holes, and Quantum Foam: A Life in Physics (Norton, New York, 1998), p. 380.

[6] J.R. Ellis, J.S. Hagelin, D.V. Nanopoulos, and M. Srednicki, Nucl. Phys. B241, 381 (1984).

[7] G. Amelino-Camelia, J.R. Ellis, N.E. Mavromatos, and D.V. Nanopoulos, Int. J. Mod. Phys. A 12, 607 (1997), hep-th/9605211.

[8] G. Amelino-Camelia, J.R. Ellis, N.E. Mavromatos, D.V. Nanopoulos, and S. Sarkar, Nature (London) 393, 763 (1998), astro-ph/9712103.

[9] L. Gonzalez-Mestres, physics/9704017.

[10] J.R. Ellis, N.E. Mavromatos, D.V. Nanopoulos, and A.S. Sakharov, gr-qc/0312044.

[11] J.R. Ellis, N.E. Mavromatos, D.V. Nanopoulos, and A.S. Sakharov, Nature (London) 428, (2004), http://dx.doi.org/ 10.1038/nature02481, astro-ph/0309144.

[12] J.R. Ellis, N.E. Mavromatos, and D.V. Nanopoulos, Phys. Rev. D 62, 084019 (2000), gr-qc/0006004.

[13] I.I. Kogan, N.E. Mavromatos, and J.F. Wheater, Phys. Lett. B 387, 483 (1996), hep-th/9606102.

[14] N.E. Mavromatos and R.J. Szabo, J. High Energy Phys. 10, 027 (2001), hep-th/0106259.

[15] J.R. Ellis, N.E. Mavromatos, and D.V. Nanopoulos, Int. J. Mod. Phys. A 13, 1059 (1998), hep-th/9609238.

[16] C. Bachas, Phys. Lett. B 374, 37 (1996), hep-th/9511043.

[17] C. Bachas and M. Porrati, Phys. Lett. B 296, 77 (1992), hep-th/9209032.

[18] G. Lifschytz, Phys. Lett. B 388, 720 (1996), hep-th/9604156.
[19] O. Bergman, M.R. Gaberdiel, and G. Lifschytz, Nucl. Phys. B509, 194 (1998), hep-th/9705130.

[20] J.H. Schwarz, hep-th/9907061.

[21] E. Bergshoeff, R. Kallosh, T. Ortin, D. Roest, and A. Van Proeyen, Class. Quantum Grav. 18, 3359 (2001), hep-th/0103233.

[22] U.H. Danielsson and G. Ferretti, Int. J. Mod. Phys. A 12, 4581 (1997), hep-th/9610082.

[23] N.E. Mavromatos and R.J. Szabo, Phys. Rev. D 59, 104018 (1999), hep-th/9808124.

[24] N.E. Mavromatos and R.J. Szabo, J. High Energy Phys. 01, 041 (2003), hep-th/0207273.

[25] V. Periwal and O. Tafjord, Phys. Rev. D 54, 3690 (1996), hep-th/9603156.

[26] J. Distler and H. Kawai, Nucl. Phys. B321, 509 (1989).

[27] J. Distler, Z. Hlousek, and H. Kawai, Int. J. Mod. Phys. A 5, 391 (1990).

[28] I. Antoniadis, C. Bachas, J.R. Ellis, and D.V. Nanopoulos, Nucl. Phys. B328, 117 (1989).

[29] J.R. Ellis, N.E. Mavromatos, and D.V. Nanopoulos, hep-th/9403133.

[30] J.R. Ellis, N.E. Mavromatos, and D.V. Nanopoulos, Phys. Lett. B 293, 37 (1992), hep-th/9207103.

[31] E. Gravanis and N.E. Mavromatos, Phys. Lett. B 547, 117 (2002), hep-th/0205298.

[32] I. Antoniadis and K. Benakli, Int. J. Mod. Phys. A 15, 4237 (2000), hep-ph/0007226.

[33] A. Hashimoto and I.R. Klebanov, Phys. Lett. B 381, 437 (1996), hep-th/9604065.

[34] E. Witten, Nucl. Phys. B460, 335 (1996), hep-th/9510135.

[35] J.R. Ellis, N.E. Mavromatos, and D.V. Nanopoulos, Phys. Rev. D 63, 124025 (2001), hep-th/0012216.

[36] M. Berkooz, M.R. Douglas, and R.G. Leigh, Nucl. Phys. B480, 265 (1996), hep-th/9606139.

[37] J.R. Ellis, N.E. Mavromatos, and E. Winstanley, Phys. Lett. B 476, 165 (2000), hep-th/9909068.

[38] J.R. Ellis, N.E. Mavromatos, and A.S. Sakharov, Astropart. Phys. 20, 669 (2004), astro-ph/0308403.

[39] J.R. Ellis, K. Farakos, N.E. Mavromatos, V.A. Mitsou, and D.V. Nanopoulos, Astrophys. J. 535, 139 (2000), astro-ph/9907340.

[40] J.R. Ellis, N.E. Mavromatos, D.V. Nanopoulos, and A.S. Sakharov, Astron. Astrophys. 402, 409 (2003), astro-ph/0210124. 